\title{
On Sumsets and Convex Hull
}

\author{
Károly J. Böröczky • Francisco Santos • \\ Oriol Serra
}

Received: 24 July 2013 / Revised: 17 August 2014 / Accepted: 18 August 2014 /

Published online: 17 September 2014

(C) Springer Science+Business Media New York 2014

\begin{abstract}
One classical result of Freiman gives the optimal lower bound for the cardinality of $A+A$ if $A$ is a $d$-dimensional finite set in $\mathbb{R}^{d}$. Matolcsi and Ruzsa have recently generalized this lower bound to $|A+k B|$ if $B$ is $d$-dimensional, and $A$ is contained in the convex hull of $B$. We characterize the equality case of the Matolcsi-Ruzsa bound. The argument is based partially on understanding triangulations of polytopes.
\end{abstract}

Keywords Sumsets $\cdot$ Shellable triangulations $\cdot h$-vector

\section{Introduction}

The topic of this paper is the cardinality of the sum of finite sets in the real affine space. For thorough surveys and background, consult Ruzsa [6], and Tao and Vu [9].

K. J. Böröczky

Alfréd Rényi Institute of Mathematics, Hungarian Academy of Sciences, Reáltanoda u. 13-15,

Budapest 1053, Hungary

e-mail: kboroczky@gmail.com

K. J. Böröczky

Central European University, Nador u. 9, Budapest 1051, Hungary

F. Santos

Departamento de Matemáticas, Estadística y Computación Universidad de Cantabria, Av. de los Castros 48, 39005 Santander, Spain

O. Serra

Departamento de Matemàtica Aplicada 4, Universitat Politècnica de Catalunya, Jordi Girona 1, 08034 Barcelona, Spain 
A set $A$ in $\mathbb{R}^{d}$ is $d$-dimensional if it is not contained in any affine hyperplane. One seminal result proved by Freiman [1] is that for any finite $d$-dimensional set $A$ in $\mathbb{R}^{d}$,

$$
|A+A| \geq(d+1)|A|-\frac{d(d+1)}{2} .
$$

The inequality is tight and the extremal sets have been characterized by Stanchescu [7]. It was recently generalized by Matolcsi and Ruzsa [5] as follows.

Theorem 1 (Matolcsi-Ruzsa) If $B$ is finite d-dimensional in $\mathbb{R}^{d}$ and $A \subset[B]$, then for every $k \in \mathbb{N}$

$$
|A+k B| \geq\left(\begin{array}{c}
d+k \\
k
\end{array}\right)|A|-k\left(\begin{array}{l}
d+k \\
k+1
\end{array}\right) .
$$

In particular, taking $A=B$ they get the following, of which (1) is the case $k=2$ :

Corollary 2 (Freiman-Matolcsi-Ruzsa) If A is finite d-dimensional in $\mathbb{R}^{d}$, then for every $k \in \mathbb{N}$

$$
|k A| \geq\left(\begin{array}{c}
d+k-1 \\
k-1
\end{array}\right)|A|-(k-1)\left(\begin{array}{c}
d+k-1 \\
k
\end{array}\right) .
$$

In these results, for a set $X \subset \mathbb{R}^{d}$, we set $1 X=X, k X=(k-1) X+X$ for $k \geq 2$, and $0 X=\{0\}$. The sum $X+\emptyset$ is always the empty set. The convex hull of the set $X \subset \mathbb{R}^{d}$ is denoted by $[X]$. Similarly, $\left[x_{1}, \ldots, x_{m}\right]$ will denote the convex hull of points $x_{1}, \ldots, x_{m} \in \mathbb{R}^{d}$.

One of the motivations of the Matolcsi-Ruzsa inequality is the observation that to prove (1) for the sumset $A+A$, the relevant points of the second summand are the vertices of $[A]$.

The goal of this paper is to give an explicit characterization of the sets $A$ and $B$ for which the inequality in Theorem 1 is tight, that is, for which $A \subset[B]$ and

$$
|A+k B|=\left(\begin{array}{c}
d+k \\
k
\end{array}\right)|A|-k\left(\begin{array}{l}
d+k \\
k+1
\end{array}\right) .
$$

We call such a pair $(A, B)$ a $k$-critical pair. As in Matolcsi and Ruzsa [5], triangulations of $B$ have a crucial role in our paper. By a triangulation $\mathcal{T}$ of $B$, we mean a triangulation of $[B]$ where the set of vertices of $\mathcal{T}$ is $B$. In addition, $\mathcal{T}$ is called stacked if it has $|B|-d$ full-dimensional simplices (which is the minimum possible number of simplices in a triangulation of $|B|$ points in $\left.\mathbb{R}^{d}\right)$. As first steps in the characterization, we show that for every $k$-critical pair $(A, B)$ :

- $B \subset A$ (Lemma 18).

- $\left(A \cap\left[B^{\prime}\right], B^{\prime}\right)$ is also $k$-critical, for any subset $B^{\prime} \subset B$ (Lemma 19).

- $B$ is totally stackable (Corollary 21 ), meaning that all of its triangulations are stacked. 
Total stackability is a very restrictive property that can be expressed in different ways (Lemma 11) and totally stackable sets are completely characterized by Nill and Padrol (see Theorem 12). Section 3 includes these results and some preliminary background on triangulations. The fact that $B$ needs to be totally stackable in order to have equality follows from the following refinement of Theorem 1 that we prove in Sect. 6 .

Theorem 3 Let $\mathcal{T}$ be a shellable triangulation of $B$ with h-vector $\left(h_{0}, \ldots, h_{d}\right)$. Let $A$ be such that $B \subset A \subset[B]$. Then,

$$
|A+k B| \geq\left(\begin{array}{c}
d+k \\
k
\end{array}\right)|A|-k\left(\begin{array}{c}
d+k \\
k+1
\end{array}\right)+\sum_{j=2}^{\min (d, k+1)} h_{j}\left(\begin{array}{c}
d+k+1-j \\
k+1-j
\end{array}\right)
$$

The $h$-vector $\left(h_{0}, h_{1}, \ldots, h_{d}\right) \in \mathbb{N}^{d+1}$ (here and in what follows $\mathbb{N}=\{0,1,2, \ldots\}$ ) of a $d$-dimensional triangulation is a classical invariant in geometric combinatorics, which can be read either from the $f$-vector (the number of simplices of each dimension) or from a shelling. See more background on this topic in Sect. 3. Since $h_{i} \geq 0$ for every $i$, Theorem 3 implies Theorem 1. But it also tells us that in order to have equality in Theorem 1 all the shellable triangulations of $B$ need to have $h_{i}=0$ for all $i \geq 2$, which is equivalent to them having $|B|-d$ simplices. Hence, $B$ needs to be totally stackable.

It is worth noticing that the inequality in Theorem 3 is equivalent to

$$
|A+k B| \geq\left(\begin{array}{c}
d+k \\
k
\end{array}\right)|A \backslash B|+\sum_{j=0}^{\min (d, k+1)} h_{j}\left(\begin{array}{c}
d+k+1-j \\
k+1-j
\end{array}\right) .
$$

For the case $A=B$ this leads to the following refinement of Corollary 2:

Corollary 4 Let $\mathcal{T}$ be a shellable triangulation of A with $h$-vector $\left(h_{0}, \ldots, h_{d}\right)$. Then,

$$
|k A| \geq \sum_{j=0}^{\min (d, k)} h_{j}\left(\begin{array}{c}
d+k-j \\
k-j
\end{array}\right)
$$

The geometric structure of critical pairs is complemented by its arithmetic structure. To express this arithmetic structure we introduce the following concepts. For finite $B \subset \mathbb{R}^{d}$, we write $\Lambda(B)$ to denote the additive subgroup of $\mathbb{R}^{d}$ generated by $B-B$, and hence by $B$ if $0 \in B$. We note that $\Lambda(B)$ is called a lattice if it is of rank $d$, which will be the typical case.

Definition We say that $A \subset[B]$ is stable with respect to $B$, or $B$-stable if

$$
(A+\Lambda(B)) \cap[B]=A .
$$

The fact that $A$ is $B$-stable provides a substantial arithmetic structure to $A$. For example, suppose that $A$ is $B$-stable and let $l$ be a line intersecting $A$ and such that $\Lambda(B)$ contains non-zero vectors parallel to $l$. Let $w$ be the shortest such vector (which 
is unique up to sign). Then $A \cap l$ can be partitioned into arithmetic progressions with common difference $w$, each of which equals $(x+\mathbb{Z} w) \cap[B]$ for some $x \in \mathbb{R}^{d}$. If, in addition, $l$ contains an edge $[u, v]$ of $[B]$, then one of these arithmetic progressions contains the vertices $u, v$ of the edge. In particular, for two parallel lines $l, l^{\prime}$ intersecting $A$ these arithmetic progressions have the same common difference ( $w$ depends only on the direction of $l$ ) and if the lines contain edges $e, e^{\prime}$ with $\ell(e) \geq \ell\left(e^{\prime}\right)$ of $B$ then the translation of $A \cap e^{\prime}$ within $e$ matching one vertex of $e$ is contained in $A \cap e$.

As usual, a $d$-dimensional (straight) prism $P$ is the Minkowski sum of a $(d-1)$-dimensional polytope $Q$ and a segment $s$ not parallel to it. Edges of $P$ parallel to $s$ are called vertical. We are interested in the case where $Q$ is a simplex and where the vertical edges are allowed to have different lengths. We abbreviate this as a simplex-prism. Put differently, a simplex prism is any polytope afffinely equivalent to

$$
\left\{(x, t) \in \mathbb{R}^{d+1}: x \in \Delta \subset \mathbb{R}^{d}, t \in \mathbb{R}, \ell(x) \leq t \leq u(x)\right\},
$$

where $\Delta$ is a $d$-simplex and $\ell, u$ are two affine functions with $\ell(x)<u(x)$ for every $x \in \Delta$.

With these geometric and arithmetic ingredients, Sects. 7, 8 and 9 lead to the following explicit characterization of the critical pairs via a case study based in the characterization of totally stackable sets.

Theorem 5 Let $k \geq 1, d \geq 1$, and let $A, B \subset \mathbb{R}^{d}$ be finite such that $B$ spans $\mathbb{R}^{d}$, and $A \subset[B]$. Equality holds in Theorem 1 if and only if $B \subset A, B$ is contained in the union of the edges of $[B]$, and one of the following conditions hold.

(i) $|B|=d+1$. That is, $B$ is the vertex set of a $d$-simplex.

(ii) For $d \geq 1, B$ consists of the vertices of the simplex $\left[v_{0}, \ldots, v_{d}\right]$, and some extra points on the edge $\left[v_{0}, v_{d}\right]$. The points of $B$ on this edge are part of an arithmetic progression $D$ contained in $A$, and $A \backslash(B \cup D)$ is the disjoint union of translates of $D \backslash\left\{v_{0}\right\}$.

(iii) For $d \geq 2$, $[B]$ is a simplex-prism, $A$ is stable with respect to $B$, and $A$ is contained in the vertical edges of $B$.

(iv) For $d=2$, A consists of the vertices of a triangle and the midpoints of its sides.

(v) For $d=2, B$ consists of the the vertices of a parallelogram, and $A$ is stable with respect to $B$ and contained in the boundary of $[B]$.

(vi) For $2 \leq q<d, A$ and $B$ are the unions of some $d-q$ points and sets $A^{\prime}, B^{\prime}$ respectively, where $\left(A^{\prime}, B^{\prime}\right)$ is a pair of q-dimensional sets of type (iii), (iv) or (v). That is to say, $[A]=[B]$ is an iterated pyramid over $\left[A^{\prime}\right]=\left[B^{\prime}\right]$ and the only points of $A \backslash A^{\prime}$ are the new vertices.

Observe that in part (vi) we do not include (iterated) pyramids over the configurations of parts (i) and (ii) because these are again configurations of the types described in (i) and (ii).

The characterization in Theorem 5 reveals two interesting facts about critical pairs.

- The characterization is independent of $k$. One direction (the fact that $k$-criticality implies $(k-1)$-criticality, if $k \geq 2)$ is proved in Lemma 22. The other direction is only proved as a consequence of the full characterization. 
- If $(A, B)$ is critical then $A$ is stable with respect to $B$. Actually, criticality of the pair $(A, B)$ depends on $A$ and the lattice $\Lambda(B)$ generated by the points of $B$ rather than the structure of $B$ itself. Again, without resorting to the full characterization, we only have a partial direct proof of this, namely the case of dimension one (Proposition 8).

In turn, Theorem 5 yields the following concerning the equality case of Corollary 2.

Corollary 6 Let $k \geq 2, d \geq 2$, and let the finite $A$ span $\mathbb{R}^{d}$. Equality holds in Corollary 2 if and only if one of the following conditions hold.

(i) The set A consists of the vertices of a simplex plus an arithmetic progression contained in an edge of the simplex, starting and ending at the endpoints of the edge.

(ii) The set A consists of the vertices of a simplex plus the midpoints of the sides of a certain 2-face of the simplex.

(iii) For $d \geq q \geq 2$, [A] is an iterated pyramid over a $q$-dimensional simplex-prism. There exists a non-zero $w \in \mathbb{R}^{d}$ such that $A$ consists of the vertices of $[A]$ and, for each vertical edge of the prism, the arithmetic progression of difference $w$ starting and ending at its endpoints.

Actually, Corollary 6 admits the more concise form of Corollary 7. In it, we say that a triangulation $\mathcal{T}$ of a finite set $A$ spanning $\mathbb{R}^{d}$ is unimodular if $\Lambda(A)$ is a lattice with determinant $\Delta$, and each full dimensional simplex of $\mathcal{T}$ has volume $\Delta / d$ !. We note that if $A$ has a stacked unimodular triangulation then all of its triangulations are unimodular and stacked.

Corollary 7 Let $k \geq 2, d \geq 2$, and let the finite $A$ span $\mathbb{R}^{d}$. Equality holds in Corollary 2 if and only if $A$ has a stacked unimodular triangulation.

To prove Theorem 5, first we consider the one-dimensional case in Sect. 2, which is the base of the arithmetic structure of critical pairs. Next we discuss some useful properties of triangulations of convex polytopes in Sect. 3. Section 4 reviews the proof of the Matolcsi-Ruzsa inequality Theorem 1, and concludes with a technical, but useful, characterization (Theorem 15) of the equality case. Based on this result, we show in Sect. 5 that the pairs $(A, B)$ listed in Theorem 5 are $k$-critical for any $k \geq 1$. Theorem 15 is also the base of the arguments leading to the fundamental properties of $k$-critical pairs in Sect. 6. Finally, a case by case analysis in Sects. 7, 8 and 9 describes explicitly the arithmetic structure of the cases in Theorem 5. In Sect. 10 we show how the results of the previous sections imply that the list in Theorem 1 is complete.

\section{The Case of Dimension One}

It is instructive to discuss the one-dimensional version of Theorem 5 first, because it does not require the geometric machinery built later on, and it provides the base of the arithmetic structure of higher dimensional critical pairs. 
For rational $0 \leq b_{1}<\cdots<b_{n}, n \geq 2$, we define $\operatorname{gcd}\left\{b_{1}, \ldots, b_{n}\right\}$ to be the largest rational number $w$ such that $b_{1} / w, \ldots, b_{n} / w$ are integers. We observe that if $A, B \subset \mathbb{R}$ are finite such that $A \subset[B]=[0,1]$, then $A$ being stable with respect to $B$ is equivalent to saying that $B \subset \mathbb{Q}$, and $A$ is the union of maximal arithmetic progressions in $[0,1]$ with difference $w=\operatorname{gcd}(B)$.

We note that the one-dimensional version of Theorem 1 reads as follows. If $A, B \subset \mathbb{R}$ are finite sets with $A \subset[B]$, and $k \geq 1$, then

$$
|A+k B| \geq(k+1)(|A|-1)+1 \text {. }
$$

The first part of the next proposition gives the one-dimensional version of Theorem 5 . The second part will be used later.

Proposition 8 Let $k \geq 1$, and let $A, B \subset \mathbb{R}$ be finite such that $A \subset[B]=[0,1]$.

(i) The pair $(A, B)$ is $k$-critical if and only if $\{0,1\} \subset A$ and $A$ is stable with respect to $B$.

(ii) If $C \subset(0,1)$ is finite, then

$$
|C+k B| \geq(k+1)|C|,
$$

with equality if and only if $C$ is stable with respect to $B$.

Proof If a finite $D \subset[B]$ is stable with respect to $B$, then

$$
D+k B=D+k\{0,1\}
$$

and hence equality holds in (3) for $D=C$, and also in (2) for $D=A$ provided that $\{0,1\} \subset A$.

We note that if either $0 \notin A$ or $1 \notin A$, then a translate of $A$ is contained in $(0,1)$, a case dealt with in (ii), which shows that $(A, B)$ is not $k$-critical. Thus let the pair $(A, B)$ be $k$-critical with $\{0,1\} \subset A$.

If $B=\{0,1\}$, then $A$ is clearly stable with respect to $B$. Therefore we may assume that $|B| \geq 3$. We write $X^{\prime}$ to denote the image of $X \subset \mathbb{R}$ in the torus $\mathbb{R} / \mathbb{Z}$ by the quotient map. In particular

$$
\left|A^{\prime}\right|=|A|-1 \text {. }
$$

Let $\widetilde{A} \subset[0,2)$ be the set obtained by choosing the smallest element of $A+B$ in each coset of $\mathbb{Z}$ intersecting $A+B$. Since $0 \in B$ yields that $\widetilde{A} \cap(A+1)=\emptyset$, the sum $A+k B$ contains the disjoint union

$$
\{k+1\} \cup \widetilde{A} \cup((A \backslash\{1\})+1) \cup \cdots \cup((A \backslash\{1\})+k) .
$$

We deduce using $\left|A^{\prime}+B^{\prime}\right| \geq\left|A^{\prime}\right|$ that

$$
|A+k B| \geq\left|A^{\prime}+B^{\prime}\right|+k\left|A^{\prime}\right|+1 \geq(k+1)\left|A^{\prime}\right|-1 \text {. }
$$


As the pair $(A, B)$ is $k$-critical, (2) and (4) yield that $\left|A^{\prime}+B^{\prime}\right|=\left|A^{\prime}\right|$. In particular

$$
a+b \in A^{\prime} \quad \text { for } a \in A^{\prime} \text { and } b \in B^{\prime} .
$$

We deduce from $|B| \geq 3$ that there exists some non-zero element of $B^{\prime}$, which in turn implies by the finiteness of $A^{\prime}$ that $B^{\prime}$ generates a finite subgroup $H$ of $\mathbb{R} / \mathbb{Z}$, and $A^{\prime}$ is the union of some cosets of $H$. It follows that $B \subset \mathbb{Q}$, and $H$ is generated by $w^{\prime}$ for $w=\operatorname{gcd}(B)$. This implies (i). The argument for (ii) is completely analogous, only $k+1 \notin C+k B$, and hence (5) is replaced by $|C+k B| \geq\left|C^{\prime}+B^{\prime}\right|+k\left|C^{\prime}\right|$ where $\left|C^{\prime}\right|=|C|$.

\section{Some Observations about Triangulations}

Throughout this paper, a triangulation of a finite point set $B \subset \mathbb{R}^{d}$ is a geometric simplicial complex with vertex set $B$ and underlying space $[B]$. A triangulation will be given as a list of $d$-simplices.

Let $\mathcal{T}=\left\{S_{1}, \ldots, S_{m}\right\}$ be a triangulation of $B$. We say that the ordering $S_{1}, \ldots, S_{m}$ of the simplices of $\mathcal{T}$ is a shelling if, for every $i$, the intersection of $S_{i}$ with $S_{1} \cup \cdots \cup S_{i-1}$ is a union of facets of $S_{i}$. Equivalently, if $S_{1} \cup \cdots \cup S_{i}$ is a topological ball for every $i$. The index of a simplex $S_{i}$ in a shelling is the number of facets of $S_{i}$ that are contained in $S_{1} \cup \cdots \cup S_{i-1}$. That is, the index of $S_{1}$ is zero and the index of every other $S_{i}$ is an integer between 1 and $d$. The $h$-vector of a shelling is the vector $h=\left(h_{0}, \ldots, h_{d}\right)$ with $h_{i}$ equal to the number of simplices of index $i$. We recall without proof some simple facts about shellings and $h$-vectors (see [4, Sect. 9.5.2] or [10, Chap. 8] for details):

Lemma 9 (i) Not every triangulation is shellable, but every point set has shellable triangulations. For example, all regular triangulations (which include placing, pulling and Delaunay triangulations) are shellable.

(ii) The h-vector of a shellable triangulation is independent of the choice of shelling. In fact, the h-vector of a (perhaps non-shellable) triangulation can be defined as

$$
h_{k}=\sum_{i=0}^{k}(-1)^{k-i}\left(\begin{array}{c}
d+1-i \\
k-i
\end{array}\right) f_{i-1},
$$

where $\left(f_{-1}, \ldots, f_{d}\right)$ is the $f$-vector of $\mathcal{T}$. That is, $f_{i}$ is the number of $i$-simplices in $\mathcal{T}$, with the convention that $f_{-1}=1$.

(iii) Every triangulation of $B$ has $h_{0}=1, h_{1}=|B|-d-1$, and $\sum h_{i}=m$, where $m$ and $d$ are the number of $d$-simplices and the dimension of $\mathcal{T}$.

One useful way of constructing triangulations of a point set is the placing procedure, which is recursively defined as follows (see [4, Sect. 4.3.1] for more details). Let $B \subset \mathbb{R}^{d}$ be a finite point set and let $x \in B$ be such that $B^{\prime}:=B \backslash\{x\}$ is $d$-dimensional and $x \notin\left[B^{\prime}\right]$. If $\mathcal{T}^{\prime}$ is a triangulation of $B^{\prime}$, we call placing of $x$ in $\mathcal{T}^{\prime}$ the triangulation $\mathcal{T}$ of $B$ obtained adding to $\mathcal{T}^{\prime}$ the pyramids with apex at $x$ of all the boundary $(d-1)$ 
-simplices of $\mathcal{T}^{\prime}$ that are visible from $x$. Here, we say that a $(d-1)$-simplex $S$ in the boundary of $B^{\prime}$ is visible from $x$ if its supporting hyperplane $H$ separates $x$ from $B^{\prime} \backslash H$. Equivalently, if $[x, y] \cap\left[B^{\prime}\right]=\{y\}$ for every point $y \in S$. It can be shown that if $\mathcal{T}^{\prime}$ is shellable then $\mathcal{T}$ is shellable too.

The placing procedure can be used to construct a (shellable) triangulation of $B$ from scratch, by choosing an initial simplex $S=\left[x_{1}, \ldots, x_{d+1}\right]$ with $\left\{x_{1}, \ldots, x_{d+1}\right\} \subset B$ and $S \cap\left(B \backslash\left\{x_{1}, \ldots, x_{d+1}\right\}\right)=\emptyset$, or to extend a given triangulation of a subset $B^{\prime} \subset B$ with $\left[B^{\prime}\right] \cap\left(B \backslash B^{\prime}\right)=\varnothing$.

We observe that if $C=\left\{x_{1}, \ldots, x_{d+1}\right\}$ is affinely independent, $s \in\{1, \ldots, d+1\}$, and $t>0$, then for the facets $F_{j}=\left[C \backslash x_{j}\right]$ of $[C], j=1, \ldots, s$, we have

$$
t \cdot\left([C] \backslash\left(\cup_{j=1}^{s} F_{j}\right)\right)=\left\{\sum_{j=1}^{d+1} \lambda_{j} x_{j}: \lambda_{j}>0 \text { for } j \leq \mathrm{s}, \forall \lambda_{j} \geq 0, \sum_{j=1}^{d+1} \lambda_{j}=t\right\} .
$$

Therefore if $k \geq 1$, and $S_{1}, \ldots, S_{m}$ is a shelling of a triangulation $\mathcal{T}$, then

$$
T_{i}+k S_{i}=(k+1) T_{i} \text { for } i=2, \ldots, m \text { and } T_{i}=S_{i} \backslash\left(S_{1} \cup \cdots \cup S_{i-1}\right) .
$$

Of special interest for us will be stacked triangulations. A stacked triangulation is one that satisfies any of the following equivalent properties, and they are a particular case of placing triangulations, hence shellable.

Lemma 10 The following properties are equivalent, for a triangulation $\mathcal{T}$ of a point set $B$.

(i) The number of $d$-simplices in $\mathcal{T}$ equals $|B|-d$.

(ii) $h_{i}=0$ for all $i \geq 2$.

(iii) The dual graph of $\mathcal{T}$ is a tree. The dual graph is the graph having as vertices the $d$-simplices of $\mathcal{T}$ and as edges the adjacent pairs (pairs that share a facet).

(iv) Every simplex of dimension at most $d-2$ of $\mathcal{T}$ is contained in $\partial[B]$.

Proof The equivalence of the first two properties follows from $\sum h_{i}=m$ and $h_{0}+$ $h_{1}=|B|-d$, where $m$ denotes the number of $d$-simplices. For a shellable triangulation $\mathcal{T}$, the implications (ii) $\Rightarrow$ (iii) $\Rightarrow$ (iv) $\Rightarrow$ (ii) are also trivial. Hence, the only thing we need to prove is that any of (i), (iii) and (iv) implies $\mathcal{T}$ to be shellable. Let the simplices in $\mathcal{T}$ be ordered $S_{1}, \ldots, S_{m}$ in such a way that $S_{i}$ shares at least one facet with $S_{1} \cup \ldots \cup S_{i-1}$, which can always be done. Then:

(i) $S_{1} \cup \cdots \cup S_{i}$ has at most one vertex more than $S_{1} \cup \cdots \cup S_{i-1}$. If the total number of vertices equals $|B|-d$ we need the number to always increase by one, which implies $\left(S_{1} \cup \cdots \cup S_{i-1}\right)$ intersects $S_{i}$ only in a facet.

(iii) If the dual graph is a tree, it has one less edge than vertices. Then, no $S_{i}$ has two facets in common with $S_{1} \cup \cdots \cup S_{i-1}$. It may in principle have a facet plus some lower dimensional face $\sigma$, but this would imply the dual graph of the link of $\sigma$ in $S_{1} \cup \cdots \cup S_{i}$ to become disconnected. Since at the end of the process all links have connected dual graphs, there has to be a $j>i$ such that $S_{j}$ also contains $\sigma$ and is glued to $S_{1} \cup \cdots \cup S_{j-1}$ along at least two facets, a contradiction. 
(iv) If every simplex of dimension at most $d-2$ of $\mathcal{T}$ is contained in $\partial[B]$, then every $(d-1)$-simplex in $\mathcal{T}$ disconnects $\mathcal{T}$. Hence the dual graph is a tree and, by the previous argument, $\mathcal{T}$ is shellable.

We call a point set $B$ totally stackable if all its triangulations are stacked. This poses heavy restrictions on the combinatorics of $B$, as we now see:

Lemma 11 Let $B \subset \mathbb{R}^{d}$ be a d-dimensional finite point set. The following conditions are equivalent:

(i) B is totally stackable.

(ii) Every $k$ points of $B$ lie in a face of $[B]$ of dimension at most $k$, for every $k$.

(iii) Every subset $C$ of at most $d-1$ points of $B$ has $[C] \subset \partial[B]$.

Proof The implication (ii) $\Rightarrow$ (iii) is obvious, and (iii) clearly implies the last property of Lemma 10 for every triangulation, hence it implies (i). So, we only need to show (i) $\Rightarrow$ (ii).

Let $C \subset B$ be a set of $k$ points and let $F$ be the minimal face of [B] containing $C$ (the carrier of $C$ ). Assume that $\operatorname{dim}(C)>k$ and, without loss of generality, that $C$ is affinely independent. It is easy to show that $B_{F}:=B \cap F$ has a triangulation $\mathcal{T}_{F}$ using $C$ as a simplex. Since $[C]$ goes through the interior of $F$, the link of $C$ in $\mathcal{T}_{F}$ is a $(\operatorname{dim}(C)-k)$-sphere. In particular, since $\operatorname{dim}(C)-k>0$, its dual graph has cycles. This $\mathcal{T}_{F}$ can be extended to a triangulation of $B$ (for example via the placing procedure, see $[4$, Section 4.3.1]) which will still have cycles in its dual graph.

Properties (ii) and (iii) have the following straightforward consequences, which will be useful in order to give an explicit description of all possible totally stackable sets:

- If $B$ is totally stackable, every point of $B$ is either a vertex of $[B]$ or lies in the relative interior of an edge of $[B]$. That is, $B$ is contained in the union of edges of $[B]$. We call the edges of $[B]$ that contain points of $B$ other than vertices loaded.

- Every subset $B^{\prime}$ of a totally stackable set $B$ is totally stackable in aff $\left(B^{\prime}\right)$.

Sets satisfying property (iii) of Lemma 11 are called of combinatorial degree one by Nill and Padrol [3], who give a complete classification of them. The description uses iterated pyramids, which we define in terms of the join operator. Let $B_{1}$ and $B_{2}$ be two finite sets in $\mathbb{R}^{d}$ whose affine hulls are of dimensions $d_{1}$ and $d_{2}$, respectively. We say that $B_{1} \cup B_{2}$ is a join of $B_{1}$ and $B_{2}$ if the affine hull of $B_{1} \cup B_{2}$ is of dimension $d_{1}+d_{2}+1$. For $i=1,2$, consider a triangulation for $B_{i}$ where the number of $d_{i}$-simplices is $m_{i}$. These two triangulations induce a triangulation for the join $B_{1} \cup B_{2}$ where the number of $\left(d_{1}+d_{2}+1\right)$-simplices is $m_{1} m_{2}$. Moreover, all triangulations of a join arise in this way. A join where $B_{2}$ is a single point is a pyramid, and if $B_{2}$ is affinely independent it is an iterated pyramid over $B_{1}$.

Theorem 12 (Nill and Padrol [3]) Let $B$ be a finite set in $\mathbb{R}^{d}$ not contained in a hyperplane. Then $B$ is totally stackable if and only if $B$ is contained in the union of the edges of $[B]$, and either of the following conditions holds. 
(i) $[B]$ is a simplex, and all loaded edges meet at a vertex.

(ii) $[B]$ is an iterated pyramid over a polygon, and every loaded edge is a side of the polygon.

(iii) $[B]$ is (projectively equivalent to) an iterated pyramid over a simplex-prism, and every loaded edge is a vertical edge of the prism.

Observe that if the polygon in case (ii) is a triangle then $[B]$ is a simplex (as in case (i)), but still the two cases differ in which edges are allowed to be loaded. In part (iii) we need to allow projective equivalence on the prisms since being stackable is invariant under it. The effect of a projective deformation on a prism is that the "vertical" edges may no longer be parallel, but rather span lines meeting at a point.

\section{A Proof of Theorem 1 and Some Consequences for Critical Pairs}

In this section, we review the proof of Theorem 1 from Matolcsi and Ruzsa [5] in order to analyze the equality case. This will lead to a technical but useful characterization of critical pairs (Theorem 15), a strengthening of the Matolcsi-Ruzsa inequality (Theorem 20), and various fundamental properties of critical pairs (Theorem 23).

Recall that $k M=M+\cdots+M$ denotes the $k$-fold Minkowski sum of $M$ with itself and $k \dot{M}=\{k x: x \in M\}$ denotes the dilation of $M$ by a factor of $k$. We note that if $M$ is a convex set in $\mathbb{R}^{d}$, and $k \geq 1$ is an integer, then

$$
k M=\{k x: x \in M\}=k \cdot M .
$$

The following simple observation will be often used.

Claim 13 Let $S=[C]$ be a d-simplex for $C=\left\{v_{0}, \ldots, v_{d}\right\}$.

(i) $|k C|=\left(\begin{array}{c}d+k \\ k\end{array}\right)$.

(ii) For distinct points $a, b \in S$ and $k \geq 1$ we have

$$
(a+k C) \cap(b+k C)=\emptyset,
$$

unless both $a, b \in C$.

Proof We may assume that $v_{0}$ is the origin, and $v_{1}, \ldots, v_{d}$ form the orthonormal basis. In this case we have

$$
k C=\left\{\left(t_{1}, \ldots, t_{d}\right) \in \mathbb{N}^{d}: \sum_{i=0}^{d} t_{i} \leq k\right\},
$$

and hence (i) follows by enumeration.

For (ii), we observe that, for each pair $x, y \in k C$ of distinct points, the sets $x+$ $[0,1)^{d}$ and $y+[0,1)^{d}$ are disjoint. Since $S \backslash\left\{v_{0}, \ldots, v_{d}\right\} \subset[0,1)^{d}$, it follows from $(a+k C) \cap(b+k C) \neq \emptyset$ that either $a$ or $b$ is a vertex, say $a$ is a vertex of $S$. Then $x+a \in \mathbb{Z}^{d}$, thus $b$ is a vertex of $S$ as well. 
Corollary 14 If $C=\left\{v_{0}, \ldots, v_{d}\right\}$ is the vertex set of a simplex and if $A \subset[C]$, then

$$
\left.|A+k C|=\left(\begin{array}{c}
d+k \\
k
\end{array}\right)|A|-\sum_{i=1}^{|A \cap C|}\left(\begin{array}{c}
d+k \\
k
\end{array}\right)-\left(\begin{array}{c}
d+k+1-i \\
k
\end{array}\right)\right) .
$$

In particular,

$$
|A+k C| \geq\left(\begin{array}{c}
d+k \\
k
\end{array}\right)|A|-k\left(\begin{array}{c}
d+k \\
k+1
\end{array}\right)
$$

with equality if and only if $C \subset A$.

Proof Clearly,

$$
A+k C=\bigcup_{a \in A}(a+k C) .
$$

By Claim 13(i) each of the sets $a+k C$ has cardinality $\left(\begin{array}{c}d+k \\ k\end{array}\right)$ and, by Claim 13(ii), they are pairwise disjoint except when $a, a^{\prime} \in C \cap A$. That is:

$$
|A+k C|=\left(\begin{array}{c}
d+k \\
k
\end{array}\right)|A \backslash C|+|(A \cap C)+k C| .
$$

To prove (i) we only need to check that

$$
|(A \cap C)+k C|=\sum_{i=1}^{|A \cap C|}\left(\begin{array}{c}
d+k+1-i \\
k
\end{array}\right)
$$

For this assume, as in the proof of Claim 13(i), that $v_{0}$ is the origin, and $v_{1}, \ldots, v_{d}$ form an orthonormal basis. Let $C_{l}:=\left\{v_{0}, v_{1}, \ldots, v_{l-1}\right\}, 1 \leq l \leq d+1$, and assume that $A \cap C=C_{t}$ for some $t$. Observe that

$$
C_{1}+k C=k C
$$

and, for each $l=2, \ldots, d+1$,

$$
\left(C_{l}+k C\right) \backslash\left(C_{l-1}+k C\right)=\left\{\left(t_{1}, \ldots, t_{d}\right) \in \mathbb{N}^{d}: \begin{array}{l}
\sum_{i=0}^{d} t_{i}=k+1 \\
t_{1}=\cdots=t_{l-2}=0 \\
t_{l-1}>0
\end{array}\right\} .
$$

Hence,

$$
\left|\left(C_{l}+k C\right) \backslash\left(C_{l-1}+k C\right)\right|=\left(\begin{array}{c}
d+k+1-l \\
k
\end{array}\right),
$$


and

$$
|(A \cap C)+k C|=\left|C_{t}+k C\right|=\sum_{i=1}^{|A \cap C|}\left(\begin{array}{c}
d+k+1-i \\
k
\end{array}\right) .
$$

For the second part of the statement, observe that in $\sum_{i=1}^{|A \cap C|}\left(\left(\begin{array}{c}d+k \\ k\end{array}\right)-\left(\begin{array}{c}d+k+1-i \\ k\end{array}\right)\right)$ each summand is non-negative, so

$$
\left(\begin{array}{c}
d+k \\
k
\end{array}\right)|A|-|A+k C| \leq \sum_{i=1}^{d+1}\left(\left(\begin{array}{c}
d+k \\
k
\end{array}\right)-\left(\begin{array}{c}
d+k+1-i \\
k
\end{array}\right)\right)=k\left(\begin{array}{l}
d+k \\
k+1
\end{array}\right)
$$

with equality if and only if $|A \cap C|=d+1$.

Proof of Theorem 1 Let $S_{1}, \ldots, S_{m}$ be a shelling of a triangulation $\mathcal{T}$ of $B$. Let $C_{i}$ be the set of vertices $S_{i}$. According to (8),

$$
(k+1) S_{i}, i=1, \ldots, m \text {, form a triangulation of }(k+1)[B] .
$$

We define

$$
\begin{aligned}
& A_{1}=A \cap S_{1}, \\
& A_{i}=A \cap\left(S_{i} \backslash\left(S_{1} \cup \ldots \cup S_{i-1}\right)\right) \quad \text { for } i=2, \ldots, m .
\end{aligned}
$$

We observe that $A_{1}, \ldots, A_{m}$ form a partition of $A$. Moreover, by shellability,

$A_{i} \subset S_{i}$, and $A_{i}$ contains at most one vertex of $S_{i}$ for $i=2, \ldots, m$.

We deduce from (7) that

$$
|A+k B| \geq \sum_{i=1}^{m}\left|A_{i}+k C_{i}\right|
$$

Now Corollary 14 yields that

$$
\left|A_{1}+k C_{1}\right| \geq\left(\begin{array}{c}
d+k \\
k
\end{array}\right)\left|A_{1}\right|-k\left(\begin{array}{l}
d+k \\
k+1
\end{array}\right)
$$

(with equality if and only if $C_{1} \subset A_{1}$ ), and Claim 13 (i) and (ii) imply by (10) that

$$
\left|A_{i}+k C_{i}\right|=\sum_{a \in A_{i}}\left|a+k C_{i}\right|=\left(\begin{array}{c}
d+k \\
k
\end{array}\right)\left|A_{i}\right| \quad \text { for } i=2, \ldots, m .
$$

Theorem 1 follows from combining (11), (12) and (13). 
Using the notation of the above proof, the following characterization of equality in Theorem 1 follows from (7) and (9) on the one hand, and (11), (12) and (13) on the other hand.

Theorem 15 Let $A, B \subset \mathbb{R}^{d}$ finite such that $A \subset[B]$ and $\operatorname{dim}[B]=d$, and let $k \geq 1$. The pair $(A, B)$ is $k$-critical if and only iffor some shelling $S_{1}, \ldots, S_{m}$ of an arbitrary triangulation $\mathcal{T}$ of $B$, we have

(i) $C_{1} \subset A$;

(ii) $A_{i}+k C_{i}=(A+k B) \cap(k+1) T_{i}$ for $i=1, \ldots, m$

where $C_{i}$ denotes the set of vertices $S_{i}, T_{1}=S_{1}$ and $T_{i}=S_{i} \backslash\left(S_{1} \cup \cdots \cup S_{i-1}\right), i \geq 2$, and $A_{i}=A \cap T_{i}$ for $i=1, \ldots, m$.

We will also use the following consequence of the proof of Theorem 1.

Lemma 16 Suppose that $A+x \subset \operatorname{int}[B]$ for some $x \in \mathbb{R}^{d}$. Then

$$
|A+k B| \geq\left(\begin{array}{c}
d+k \\
k
\end{array}\right)|A| .
$$

Proof If $A+x \subset \operatorname{int}[B]$ for some $x \in \mathbb{R}^{d}$, then $A \cap B=\emptyset$ can be assumed, and hence Corollary 14 gives, in the notation of the proof of Theorem $1,\left|A_{1}+k C_{1}\right| \geq\left(\begin{array}{c}d+k \\ k\end{array}\right)\left|A_{1}\right|$. Therefore (11) and (13) yields (14).

\section{Proof of Sufficiency in Theorem 5}

Based on Theorem 15, we show that the pairs $(A, B)$ in Theorem 5 are $k$-critical for any $k \geq 1$. First we show that we can restrict $B$ to the vertices of $[B]$ in the case of the pairs $(A, B)$ listed in Theorem 5 .

Lemma 17 If $(A, B)$ is any of the pairs listed in Theorem 5, and $B^{\prime}$ is the vertex set of $[B]$, then

$$
A+k B=A+k B^{\prime} \text { for any } k \geq 1 \text {. }
$$

Proof It is sufficient to prove that for any $a \in A$ and $b \in B$, there exist $a^{\prime} \in A$ and $b^{\prime} \in B^{\prime}$ such that $a+b=a^{\prime}+b^{\prime}$. Since $B \subset A$, we may assume that $a, b \notin B^{\prime}$. In particular, we may assume that one of the cases (ii)-(iv) holds.

Write $\{a, b\}=\{\tilde{a}, \tilde{b}\}$, where $a=\tilde{a}$ and $b=\tilde{b}$ in the cases (ii) and (iv), and vertical edge of $[B]$ containing $\tilde{b}$ is not longer than the vertical edge containing $\tilde{a}$ in the case (iii). The fact that $A$ is stable with respect to $B$ and the conditions in (ii)-(iv) of Theorem 5 mean that $\tilde{b}$ belongs to an arithmetic progression $D$ along an edge $[u, v]$ of $[B]$ containing its two vertices $u, v$, and $\tilde{a}$ belongs to $x+D \backslash\{u\} \subset A$ for some $x$. The result follows since $D+D \backslash\{u\}=\{u, v\}+D \backslash\{u\}$.

Proof of Sufficiency in Theorem 5 Let us assume that the pair $(A, B)$ satisfies one of the conditions (i)-(vi) in Theorem 5. 
If $[B]$ is a simplex, then combining Lemma 17 and Corollary 14 yields equality in Theorem 1. This covers the cases (i), (ii) and (iv) of Theorem 5, and the part of case (vi) when the pair $(A, B)$ is obtained by adding $d-2$ points to a pair $\left(A^{\prime}, B^{\prime}\right)$ described in the case (iv).

Therefore we assume that $[B]$ is an iterated pyramid over a $q$-dimensional simplexprism $P, 2 \leq q \leq d$, and referring to Lemma 17, also that $B$ consists of the vertices of $[B]$. Let $B_{0}=B \backslash(B \cap P)$. We write $v_{1}, \ldots, v_{q}, w_{1}, \ldots, w_{q}$ to denote the vertices of $P$ in a way such that the vectors $w_{i}-v_{i}$ are parallel pointing into the same direction for $i=1, \ldots, q$. We define $S_{i}=\left[\left\{v_{1}, \ldots, v_{i}, w_{i} \ldots w_{q}\right\} \cup B_{0}\right]$ for $i=1, \ldots, q$, and hence $S_{1}, \ldots, S_{q}$ form a shelling of the corresponding triangulation of $B$. We write $A_{i}, C_{i}, T_{i}$ to denote the corresponding sets defined in Theorem 15 for $i=1, \ldots, q$.

Let $k \geq 1$ and $i \in\{1, \ldots, q\}$. We claim that assuming $v_{i}=0$, we have

$$
\begin{aligned}
A+k B & =\left(A_{i}+\Lambda(B)\right) \cap(k+1)[B] \\
A_{i}+k C_{i} & =\left(A_{i}+\Lambda(B)\right) \cap(k+1) T_{i} .
\end{aligned}
$$

Before proving (15) and (16), we point out that they readily yield Theorem 15 (ii) for the shelling $S_{1}, \ldots, S_{q}$. Since Theorem 15 (i) holds by $B \subset A$, we deduce equality in Theorem 1 for the pair $(A, B)$.

To verify (15) and (16), we observe that each coset of $\Lambda(B)$ intersecting $A$ has a representative in $A_{i}$. In other words,

$$
A+\Lambda(B)=A_{i}+\Lambda(B)
$$

We distinguish two cases. If $P$ is parallelogram, then $P$ is actually a fundamental parallelogram for the two-lattice $\Lambda(B) \cap \operatorname{lin} P$. Since $A$ is stable with respect to $\Lambda(B)$, we deduce (15) by (17). In addition (16) follows from (7), and the fact that the non-zero elements of $C_{i}$ form a $\mathbb{Z}$-basis of $\Lambda(B)$. This covers the case (v) of Theorem 5 , and the corresponding part of the case (vi).

Finally we consider the case (iii) of Theorem 5, and the corresponding part of the case (vi). We assume that $P$ is not a parallelogram, and hence $A \cap P$ is contained in the vertical edges $\left[v_{j}, w_{j}\right]$ of $P, j=1, \ldots, q$. Let

$$
\left\{z_{1}, \ldots, z_{d-1}\right\}=B_{0} \cup\left(\left\{v_{1}, \ldots, v_{q}\right\} \backslash\left\{v_{i}\right\}\right),
$$

thus there exists $w \in \mathbb{R}^{d}$ pointing into the same direction as $w_{i}-v_{i}$ such that $\left\{w, z_{1}, \ldots, z_{d-1}\right\}$ form a $\mathbb{Z}$-basis for $\Lambda(B)$. It follows that there exist integers $m_{1}, \ldots, m_{q} \geq 1$ such that $w_{j}-v_{j}=m_{j} w$ for $j=1, \ldots, q$, and there exists $\Omega \subset[0,1)$ such that $v_{j}+t w \in A$ for $j \in\{1, \ldots, q\}$ and $t \in\left[0, m_{j}\right]$ if and only if $t-\lfloor t\rfloor \in \Omega$. We define the integers $n_{1}, \ldots, n_{d-1}$ by $n_{p}=m_{j}$ if $z_{p}=m_{j}$, and $n_{p}=0$ if $z_{p} \in B_{0}$. Writing

$$
\Xi=\left\{\left(i_{1}, \ldots, i_{d-1}\right) \in \mathbb{Z}^{d-1}: i_{1}+\cdots+i_{d-1}=k \text { and } i_{j} \geq 0\right\},
$$


we deduce (15) from (17) and

$$
\begin{aligned}
A+k B & =\bigcup_{\left(i_{1}, \ldots, i_{d-1}\right) \in \Xi}\left\{t w+\sum_{j=1}^{d-1} i_{j} z_{j}: t-\lfloor t\rfloor \in \Omega \text { and } 0 \leq t \leq \sum_{j=1}^{d-1} i_{j} n_{j}\right\} \\
& =\left(A_{i}+\Lambda(B)\right) \cap(k+1)[B] .
\end{aligned}
$$

Turning to (16), we observe that $C_{i} \backslash\left\{v_{i}\right\}$ form a basis for $\mathbb{R}^{d}$. Combining this fact with (7) yields

$$
A_{i}+k C_{i}=\left(A_{i}+\Lambda\left(C_{i}\right)\right) \cap(k+1) T_{i} .
$$

Since $\{w\} \cup\left(C_{i} \backslash\left\{v_{i}, w_{i}\right\}\right)$ form a $\mathbb{Z}$-basis for $\Lambda(B)$, and $A_{i}+w \subset A_{i}+\Lambda\left(C_{i}\right)$, we deduce that $A_{i}+\Lambda\left(C_{i}\right)=A_{i}+\Lambda(B)$. We conclude (16), and in turn that equality holds in Theorem 1 for the pairs $(A, B)$ in Theorem 5 .

\section{Basic Properties of $k$-Critical Pairs}

The goal of the section is to prove Theorem 23 listing some fundamental properties of $k$-critical pairs. The first one is a direct consequence of Theorem 15.

Lemma 18 If $(A, B)$ is a $k$-critical pair, $k \geq 1$, then $B \subset A$.

Proof For any $x \in B$, we consider a shellable triangulation $\mathcal{T}$ with $x \in C_{1}$ for the first simplex $S_{1}=\left[C_{1}\right]$ of $\mathcal{T}$ (this can be achieved, for example, via a placing triangulation). Theorem 15 yields $C_{1} \subset A$, thus $x \in A$.

Based on Theorem 15, we prove that criticality is preserved by taking subsets of $B$.

Lemma 19 Let $A, B$ be d-dimensional point sets with $A \subset[B]$, and let $k \geq 1$. If $(A, B)$ is $k$-critical, then $\left(A \cap\left[B^{\prime}\right], B^{\prime}\right)$ is also $k$-critical for every $B^{\prime} \subset B$.

Proof Let $\widetilde{B}=B \cap\left[B^{\prime}\right]$ and $\widetilde{A}=A \cap\left[B^{\prime}\right]$. Since $B^{\prime} \subset \widetilde{B}$ and $\left[B^{\prime}\right]=[\widetilde{B}]$, it is sufficient to prove that $(\widetilde{A}, \widetilde{B})$ is $k$-critical.

If $\operatorname{dim}\left[B^{\prime}\right]=d$, then constructing a placing triangulation first for $\widetilde{B}$, we obtain some shelling $S_{1}, \ldots, S_{m}$ of a triangulation of $B$ such that the union of $S_{1}, \ldots, S_{n}$ is $\left[B^{\prime}\right]$ for some $n \leq m$. Now Theorem 15 (i) and (ii) for the pair $(A, B)$ readily yield the analogous properties for the pair $(\widetilde{A}, \widetilde{B})$.

Next we assume that $\operatorname{dim}\left[B^{\prime}\right]=q<d$. We choose $x_{1}, \ldots, x_{d-q} \in B$ such that for $B^{*}=\left\{x_{1}, \ldots, x_{d-q}\right\} \cup \widetilde{B}$, we have $\operatorname{dim}\left[B^{*}\right]=d$ and $B \cap\left[B^{*}\right]=B^{*}$. Let $A^{*}=A \cap\left[B^{*}\right]$. We observe that $L=$ aff $B^{\prime}$ is a supporting $q$-plane to $\left[B^{*}\right]$, with $\widetilde{A}=A^{*} \cap L, \widetilde{B}=B^{*} \cap L$ and $[\widetilde{B}]=\left[B^{*}\right] \cap L$.

Let $\widetilde{S}_{1}, \ldots, \widetilde{S}_{n}$ be a shelling of some triangulation of $\widetilde{B}$, and let $\widetilde{A}_{i}, \widetilde{C}_{i}, \widetilde{T}_{i}$ for $i=1, \ldots, n$ be the corresponding sets for Theorem 15 . We need to prove that they satisfy Theorem 15 . Since $B \subset A$ according to Lemma 18, Theorem 15 (i) readily follows, and all we have to verify is Theorem 15 (ii). 
To achieve that, we observe that $S_{1}, \ldots, S_{n}$ is a shelling of a triangulation of $B^{*}$ where $S_{i}=\left[x_{1}, \ldots, x_{d-q}, \widetilde{S}_{i}\right]$ for $i=1, \ldots, n$. Writing $A_{i}, C_{i}$ and $T_{i}$ to denote the corresponding sets in Theorem 15, we have $A_{1}=\widetilde{A}_{1} \cup\left\{x_{1}, \ldots, x_{d-q}\right\}, A_{i}=\widetilde{A}_{i}$ for $i=2, \ldots, n$, moreover $\widetilde{C}_{i}=C_{i} \cap L$ and $\widetilde{T}_{i}=T_{i} \cap L$ for $i=1, \ldots, n$. The pair $\left(A^{*}, B^{*}\right)$ is $k$-critical by the $\operatorname{argument}$ above because $B^{*} \subset B$ with $\operatorname{dim} B^{*}=d$, and hence $A_{i}, C_{i}$ and $T_{i}$ satisfy Theorem 15 (ii). It follows that the same conclusion holds for $\widetilde{A}_{i}, \widetilde{C}_{i}$ and $\widetilde{T}_{i}$, as for $i=1, \ldots, n$, we have

$$
(\widetilde{A}+k \widetilde{B}) \cap \widetilde{T}_{i}=L \cap\left(\left(A^{*}+k B^{*}\right) \cap T_{i}\right) \subset L \cap\left(A_{i}+k C_{i}\right)=\widetilde{A}_{i}+k \widetilde{C}_{i},
$$

where the first and the last equality is a consequence of the fact that $L$ is a supporting $q$-plane to $\left[B^{*}\right]$.

Under the assumption $B \subset A$, the ideas in the proof of Theorem 1 also lead to a proof of Theorem 3 . We recall its statement for the convenience of the reader.

Theorem 20 Let $A, B \subset \mathbb{R}^{d}$ be finite such that $\operatorname{dim}[B]=d$ and $B \subset A \subset[B]$, and let $\mathcal{T}$ be a shellable triangulation of $B$ with $h$-vector $\left(h_{0}, \ldots, h_{d}\right)$. Then

$$
|A+k B| \geq\left(\begin{array}{c}
d+k \\
k
\end{array}\right)|A|-k\left(\begin{array}{c}
d+k \\
k+1
\end{array}\right)+\sum_{j=2}^{\min (d, k+1)} h_{j}\left(\begin{array}{c}
d+k+1-j \\
k+1-j
\end{array}\right)
$$

Proof Let $S_{1}, \ldots, S_{m}$ be a shelling of $\mathcal{T}$. We keep the same notation for $A_{i}, C_{i}$ and $T_{i}$ as in the statement of Theorem 15. In particular, we have

$$
|A+k B|=\sum_{i=1}^{m}\left|(A+k B) \cap(k+1) T_{i}\right|
$$

Let $A_{i}^{\prime}=A_{i} \backslash C_{i}$. In each $(k+1) T_{i}$, we have, by the same argument as in Corollary 14 , and taking into account that $C_{i} \subset B \subset A$,

$$
\left|(A+k B) \cap(k+1) T_{i}\right| \geq\left|A_{i}+k C_{i}\right|=\left|A_{i}^{\prime}+k C_{i}\right|+\left|(k+1) C_{i} \cap(k+1) T_{i}\right| .
$$

The first summand is

$$
\left|A_{i}^{\prime}+k C_{i}\right|=\left(\begin{array}{c}
d+k \\
k
\end{array}\right)\left|A_{i}^{\prime}\right|
$$

by Claim 13 (i), and so

$$
\sum_{i=1}^{m}\left|A_{i}^{\prime}+k C_{i}\right|=\left(\begin{array}{c}
d+k \\
k
\end{array}\right)|A \backslash B|
$$

For the second summand, let $s_{i}$ be the index of $S_{i}$ in the shelling, and hence (6) and enumeration yield 


$$
\left|(k+1) C_{i} \cap(k+1) T_{i}\right|= \begin{cases}\left(\begin{array}{c}
d+k+1-s_{i} \\
k+1-s_{i}
\end{array}\right) & \text { if } s_{i} \leq k+1 \\
0 & \text { otherwise }\end{cases}
$$

Put differently,

$$
\sum_{i=1}^{m}\left|(k+1) C_{i} \cap(k+1) T_{i}\right|=\sum_{j=0}^{\min (d, k+1)} h_{j}\left(\begin{array}{c}
d+k+1-j \\
k+1-j
\end{array}\right) .
$$

Since $(k+1) C_{i}$ and $A_{i}^{\prime}+k C_{i}$ are disjoint by Claim 13 (ii), we obtain

$$
\begin{aligned}
|A+k B| \geq & \sum_{i=1}^{m}\left|A_{i}^{\prime}+k C_{i}\right|+\sum_{i=1}^{m}\left|(k+1) C_{i} \cap(k+1) T_{i}\right| \\
= & \left(\begin{array}{c}
d+k \\
k
\end{array}\right)|A \backslash B|+\sum_{j=0}^{\min (d, k+1)} h_{j}\left(\begin{array}{c}
d+k+1-j \\
k+1-j
\end{array}\right) \\
= & \left(\begin{array}{c}
d+k \\
k
\end{array}\right)|A|+\left(\begin{array}{c}
d+k+1 \\
k+1
\end{array}\right)-(d+1)\left(\begin{array}{c}
d+k \\
k
\end{array}\right) \\
& +\sum_{j=2}^{\min (d, k+1)} h_{j}\left(\begin{array}{c}
d+k+1-j \\
k+1-j
\end{array}\right) \\
= & \left(\begin{array}{c}
d+k \\
k
\end{array}\right)|A|-k\left(\begin{array}{c}
d+k \\
k+1
\end{array}\right)+\sum_{j=2}^{\min (d, k+1)} h_{j}\left(\begin{array}{c}
d+k+1-j \\
k+1-j
\end{array}\right),
\end{aligned}
$$

where, in the last step, we use $h_{0}=1$ and $h_{1}=|B|-d-1$.

Corollary 21 If the pair $(A, B)$ is $k$-critical with $\operatorname{dim}[B]=d$, then $B$ is totally stackable. In particular, $B$ is contained in the union of the edges of $[B]$.

Proof We have $B \subset A$ according to Lemma 18 . Theorem 20 yields that every shellable (in particular, every regular) triangulation has $h_{2}=0$. According to the characterization of the $h$-vectors by Stanley [8] (see also Theorem 8.34 in Ziegler [10]), we have $h_{j}=0$ for $j \geq 2$, which, by Lemma 10 , implies that $B$ is stacked. That is, every regular triangulation of $B$ has $|B|-d d$-simplices. It is a fact (see [4, Theorem 8.5.19]) that then all the triangulations (regular or not) have the same number $|B|-d$ of $d$-simplices. That is, $B$ is totally stackable.

Next we prove that equality in Theorem 1 is preserved under reducing the value of $k$.

Lemma 22 If $(A, B)$ is $k$-criticalfor $k \geq 2$ with $\operatorname{dim}[B]=d$, then it is also $k^{\prime}$-critical for every $k^{\prime}=1, \ldots, k-1$.

Proof Let $S_{1}, \ldots, S_{m}$ be a shelling of a triangulation of $B$. We use the notation of Theorem 15. Condition (i) of Theorem 15 is independent of $k$, and hence we need to check condition (ii). 
Let $z \in(A+(k-1) B) \cap T_{i}$ for $i=1, \ldots, m$, and what we need to show is that $z \in A_{i}+(k-1) C_{i}$. Since $B$ is totally stackable by Corollary 21, we may assume that $C_{i}=\left\{v_{0}, \ldots, v_{d}\right\}$ in a way such that $T_{i}=S_{i} \backslash\left[v_{1}, \ldots, v_{d}\right]$ if $i \geq 2$, and $z \notin\left[v_{1}, \ldots, v_{d}\right]$ if $i=1$. In particular, $v_{0} \in A_{i}$, and

$$
z=\sum_{j=0}^{d} \lambda_{j} v_{j} \text { where } \lambda_{0}>0, \lambda_{j} \geq 0 \text { for } j=1, \ldots, d, \text { and } \lambda_{0}+\cdots+\lambda_{d}=k
$$

by (6). If $z \in k C_{i}$, or equivalently each $\lambda_{i}$ is an integer, then $v_{0} \in A_{i}$ and $\lambda_{0}>0$ yield that $z \in A_{i}+(k-1) C_{i}$. Therefore we assume that $z \notin k C_{i}$.

Since $z+v_{0} \in(A+k B) \cap(k+1) T_{i}$ and $(A, B)$ is $k$-critical, Theorem 15 (ii) yields that

$$
z+v_{0}=a+\sum_{j=0}^{d} m_{j} v_{j}
$$

where $a \in A_{i}$, every $m_{j} \geq 0$ is an integer. and $m_{0}+\cdots+m_{d}=k$. We have

$$
a=\sum_{j=0}^{d} \alpha_{j} v_{j} \text { where } \alpha_{0}>0, \alpha_{j} \geq 0 \text { for } j=1, \ldots, d, \text { and } \alpha_{0}+\cdots+\alpha_{d}=1
$$

and $\alpha_{0}>0$ is a consequence of (6). As $C_{i}$ is affinely independent, the coefficients satisfy $\lambda_{0}+1=\alpha_{0}+m_{0}$ and $\lambda_{j}=\alpha_{j}+m_{j}$ for $j=1, \ldots, d$.

Since $z \notin k C_{i}$, we deduce that some $\alpha_{j}$ is not integer, which in turn implies that $\alpha_{0}<1$. We have $\alpha_{0}+m_{0}=\lambda_{0}+1>1$ based on (19), thus $m_{0} \geq 1$ by $\alpha_{0}<1$. Therefore

$$
z=a+\left(m_{0}-1\right) v_{0}+\sum_{j=1}^{d} m_{j} v_{j} \in A_{i}+(k-1) C_{i}
$$

as it is required by Theorem 15 (ii).

We summarize Lemmas 18, 19 and 22 and Corollary 21 as follows.

Theorem 23 If the pair $(A, B)$ is $k$-critical for $k \geq 2$ with $\operatorname{dim}[B]=d$, then

(i) $B \subset A$;

(ii) $\left(A \cap\left[B^{\prime}\right], B^{\prime}\right)$ is also $k$-critical for every $B^{\prime} \subset B$;

(iii) $B$ is totally stackable, thus $B$ is contained in the union of the edges of $[B]$;

(iv) $(A, B)$ is 1-critical, and hence $|A+B|=(d+1)|A|-d(d+1) / 2$.

From now on we consider $k$-critical pairs $(A, B)$ for $k=1$, which will be simply called critical pairs. Theorem 23 (iv) shows that $k$-critical pairs are critical. We also speak about critical sets in the case of the one dimensional version $|A+B| \geq 2|A|-1$ of the Matolcsi-Ruzsa inequality. 


\section{The Case of a Simplex}

In this section we consider the case where $[B]$ is a $d$-simplex. First we discuss iterated pyramids, a case that will be used later on as well.

Lemma 24 Let $1 \leq q<d$, and let $(A, B)$ be a critical pair with $\operatorname{dim}[B]=d$ such that $[B]$ is an iterated pyramid over $\left[B_{0}\right]$ where $B_{0} \subset B$ and $\operatorname{dim}\left[B_{0}\right]=q$. Then

(i) $\left(A \cap\left[B_{0}\right], B_{0}\right)$ is a critical pair, and

(ii) $\left|(A \cap L)+B_{0}\right|=(q+1)|A \cap L|$ for any affine $q$-plane $L$ parallel to $L_{0}=\operatorname{aff}\left(B_{0}\right)$ intersecting $A$ and avoiding the vertices of $[B]$.

Proof We have $B \subset A$ by Theorem 23 (i), and the pair $\left(A \cap\left[B_{0}\right], B_{0}\right)$ is critical by Theorem 23 (ii). Let $[B]=\left[x_{1}, \ldots, x_{d-q}, B_{0}\right]$, and let $\widetilde{B}$ be the join of $\left\{x_{1}, \ldots, x_{d-q}\right\}$ and $B_{0}$. In particular, $(A, \widetilde{B})$ is a critical pair, by Lemma 19 .

We may assume that $0 \in B_{0}$. We divide $A$ into equivalence classes according to the cosets of $H=\mathbb{Z} x_{1}+\cdots+\mathbb{Z} x_{d-q}+L_{0}$, and hence adding $\widetilde{B} \subset H$ to different equivalence classes results in disjoint sets. One equivalent class is $\widetilde{A}=\left\{x_{1}, \ldots, x_{d-q}\right\} \cup\left(A \cap\left[B_{0}\right]\right)$, and

$$
|\widetilde{A}+\widetilde{B}| \geq(d+1)|\widetilde{A}|-d(d+1) / 2
$$

by Theorem 1 . Any other equivalence class is of the form $A \cap L$ for an affine $q$-plane $L$ parallel to $L_{0}$ that avoids $\widetilde{B}$ and intersects $A$. Since a translate of $A \cap L$ is contained in the relative interior of $\left[B_{0}\right]$, Lemma 16 yields

$$
|(A \cap L)+\widetilde{B}| \geq(d+1)|A \cap L| .
$$

As $(A, \widetilde{B})$ is a critical pair, (20) and (21) imply

$$
(d+1)|A \cap L|=|(A \cap L)+\widetilde{B}|=\left|(A \cap L)+B_{0}\right|+\sum_{i=1}^{d-q}\left|(A \cap L)+x_{i}\right|,
$$

therefore $\left|(A \cap L)+B_{0}\right|=(q+1)|A \cap L|$.

We recall that an edge of $[B]$ is loaded if it contains at least three points of $B$.

Proposition 25 Let $A, B$ be finite d-dimensional sets in $\mathbb{R}^{d}, d \geq 2$ with $A \subset[B]$. If $[B]$ is a simplex, and $(A, B)$ is a critical pair, then $B \subset A, B$ is contained in the edges of $[B]$, and one of the following conditions hold:

(i) $|B|=d+1$; or

(ii) there is a unique loaded edge $[u, v]$ of $B$; the points of $B$ in this edge are part of an arithmetic progression $D$ contained in $A$; and $A \backslash(B \cup D)$ is the disjoint union of translates $D \backslash\{v\}$; or

(iii) there exist two or three loaded edges for $B$, which are sides of a two dimensional face $T$ of $[B]$, and A consists of the vertices of $[B]$, and the midpoints of the sides of $T$. 
Proof The facts that $B \subset A$ and $B$ is contained in the edges of [B] follow from Theorem 23 (i) and (iii). Let $B^{\prime}$ be the vertex set of $[B]$.

We may assume $|B|>d+1$, and hence there exists a loaded edge $[u, v]$ of $[B]$.

It follows from Lemma 24 (i) and Proposition 8 (i) that $B \cap[u, v]$ is part of a maximal arithmetic progression $D$ containing the vertices $u, v$, and $A \cap[u, v]$ contains $D$, and the rest of $A \cap[u, v]$ is the disjoint union of translates of $D \backslash\{v\}$. In particular, Lemma 24 (ii) and Proposition 8 (ii) imply that

$$
A \backslash\left(D \cup B^{\prime}\right) \text { is the disjoint union of translates of } D \backslash\{v\} \text {. }
$$

If there exists a unique loaded edge of $B$, then (22) yields (ii). Therefore we may assume that there are at least two loaded edges of $[B]$. Since $B$ is totally stackable by Theorem 23 (iii), it follows that either all loaded edges meet in a vertex, or they form a triangular 2-dimensional face by Theorem 12 .

Therefore we may assume $\left[v_{0}, v_{1}\right]$ and $\left[v_{0}, v_{2}\right]$ are two loaded edges of $B$ with $v_{0}=0$, and let $T=\left[v_{0}, v_{1}, v_{2}\right]$ be the 2-face containing these two edges. In particular, ( $A \cap T, B \cap T)$ is a critical pair by Theorem 23 (ii). It follows by (22) that for $i=1,2$, $A \cap T$ contains an arithmetic progression $D_{i}$ of length $m_{i} \geq 3$ with endpoints $v_{0}$ and $v_{i}$. According to (22), $a_{1}=\frac{m_{1}-2}{m_{1}-1} v_{1}$ is part of a translate of $D_{2} \backslash\left\{v_{2}\right\}$ contained in $A \cap T$, and hence also of a segment $\sigma \subset T$ of length at least $\frac{m_{2}-2}{m_{2}-1}\left\|v_{2}\right\|$. Since $\frac{m_{i}-2}{m_{i}-1} \geq \frac{1}{2}$, we deduce that $m_{1}=m_{2}=3, D_{1}=\left\{v_{0}, a_{1}, v_{1}\right\}$ and $D_{2}=\left\{v_{0}, a_{2}, v_{1}\right\}$ for $a_{2}=\frac{1}{2} v_{2}$. It follows by (22) that $a_{0}=\frac{1}{2}\left(v_{1}+v_{2}\right)=a_{1}+a_{2} \in A$.

Let $a \in A \backslash\left(D_{1} \cup B^{\prime}\right)$, and let $L=a+\operatorname{lin}\left\{v_{1}, v_{2}\right\}$. It follows by (22) applied to the edge $\left[v_{0}, v_{1}\right]$ that $a \in\left\{p, p-a_{1}\right\}$ where $\left\{p, p-a_{1}\right\} \subset A$. Now $p \notin\left(D_{2} \cup B^{\prime}\right)$, and hence applying (22) to the edge $\left[v_{0}, v_{2}\right]$, we conclude that either $p+a_{2} \in A$, or $p-a_{2} \in A$. In other words, either $\left[p, p-a_{1}, p+a_{2}\right] \subset[B] \cap L$, or $\left[p, p-a_{1}, p-a_{2}\right] \subset$ $[B] \cap L$. Since $[B] \cap L$ is a translate of $\lambda T$ for $\lambda \in(0,1]$ and $\left\{p, p-a_{1}\right\} \cap D_{1}=\emptyset$, we deduce that $\lambda=1$, and $\left\{p, p-a_{1}, p-a_{2}\right\}=\left\{a_{0}, a_{2}, a_{1}\right\}$. Therefore $A$ consists of the vertices of $[B]$, and the midpoints of $T$.

\section{Critical Pairs $(A, B)$ with $\operatorname{dim}[B]=2$}

In this section, $A, B$ are finite sets in $\mathbb{R}^{2}$ satisfying that $A \subset[B]$ and $B$ spans $\mathbb{R}^{2}$. Thus the case $k=1$ of Theorem 1 can be written into the form

$$
|A+B| \geq 3|A|-3 .
$$

We note that the case when $[B]$ is a triangle is handled in Sect. 7, and hence we start with the case when $[B]$ is a quadrilateral.

Proposition 26 Let $(A, B)$ be a critical pair with $[B]=\left[v_{0}, v_{1}, v_{2}, v_{3}\right]$ a quadrilateral. Then $A \subset \partial[B]$ and $[B]$ is a trapezoid. Moreover

(i) if $B$ consists of the vertices of a parallelogram, then $A$ can be partitioned into pairs of points, each a translate of a pair of consecutive points of $B$; 
(ii) if $B$ has a loaded edge or is not a parallelogram, then $A$ is contained in two parallel edges of $B$, say $e_{1}=\left[v_{0}, v_{1}\right]$ and $e_{2}=\left[v_{2}, v_{3}\right]$, and each of $A \cap e_{i}$ can be partitioned into maximal arithmetic progressions with common difference $w$; in addition, if $\ell\left(e_{2}\right) \leq \ell\left(e_{1}\right)$ and $\left[v_{0}, v_{2}\right]$ is an edge of $[B]$, then $\left(A \cap e_{2}\right)-\left(v_{2}-v_{0}\right)=$ $A \cap\left(e_{2}-\left(v_{2}-v_{0}\right)\right)$.

Proof Let $B^{\prime}=\left\{v_{0}, v_{1}, v_{2}, v_{3}\right\}$ be the vertices of $B$, where we may assume that $v_{0}=o$ and $\left[0, v_{1}\right],\left[0, v_{2}\right]$ are edges of $[B]$. By Theorem 23, we may assume that $B \subset A$ and that $\left(A, B^{\prime}\right)$ is critical.

Suppose that $[B]$ has no pair of parallel sides. We say that a side of $[B]$ is big if the sum of the angles at the endpoints of the side is less than $\pi$. Since one side out of two opposite sides of $[B]$ is big, there exists a vertex of $[B]$ where two big sides meet. Therefore we may assume that $B=\left\{o, v_{1}, v_{2}, t_{1} v_{1}+t_{2} v_{2}\right\}$, where

$$
0<t_{1} \leq t_{2}<1 \text { and } t_{1}+t_{2}>1
$$

In addition, if $s_{1} v_{1}+s_{2} v_{2} \in A$ then $s_{1}+s_{2} \leq t_{1}+t_{2}$.

Let $B_{0}^{\prime}=\left\{o, v_{1}, v_{2}\right\}$. We observe that any coset of $\mathbb{Z}^{2}=\mathbb{Z} v_{1}+\mathbb{Z} v_{2}$ intersects $[0,1) v_{1}+[0,1) v_{2}$ in exactly one point, therefore no two points of $A \backslash B_{0}^{\prime}$ are in the same coset. We deduce that

$$
\left|A+B_{0}^{\prime}\right|=\left|A \backslash B_{0}^{\prime}\right| \cdot\left|B_{0}^{\prime}\right|+\left|2 B_{0}^{\prime}\right|=3(|A|-3)+6=3 \cdot|A|-3 .
$$

On the other hand, if $s_{1} v_{1}+s_{2} v_{2} \in A+B_{0}$ then $s_{1}+s_{2} \leq t_{1}+t_{2}+1$. Therefore $2\left(t_{1}+t_{2}\right)>t_{1}+t_{2}+1$ yields

$$
2\left(t_{1} v_{1}+t_{2} v_{2}\right) \in\left(A+B^{\prime}\right) \backslash\left(A+B_{0}^{\prime}\right),
$$

and hence $\left|A+B^{\prime}\right|>\left|A+B_{0}^{\prime}\right|=3 \cdot|A|-3$, contradicting that $\left(A, B^{\prime}\right)$ is a critical pair. This proves the first part of the statement.

For (i), suppose that $B=B^{\prime}$ and $[B]$ is a parallelogram. We may assume that $[B]=[0,1]^{2}$. We partition $A=A_{0} \cup \cdots \cup A_{t}$ into equivalence classes according to $\mathbb{Z}^{2}$, where $A_{0}=B$. We observe that, for $i>0$, each $A_{i}$ consists either of one single point or a pair which is a translate of a pair of consecutive vertices in $B$. We have

$$
|A+B|=\sum_{i=0}^{k}\left|A_{i}+B\right|=3\left|A_{0}\right|-3+\sum_{i=1}^{k}\left|A_{i}+B\right|=3|A|-3,
$$

which implies $\left|A_{i}+B\right|=3\left|A_{i}\right|$ for each $i=1, \ldots, k$. This implies that no $A_{i}$ consists of a single point.

Finally, to prove (ii), we suppose that $v_{1}=\lambda\left(v_{3}-v_{2}\right)$ with $\lambda \geq 1$ and that the edge $e_{1}=\left[o, v_{1}\right]$ is loaded if $\lambda=1$. Set $A_{i}=A \cap e_{i}$ and $B_{i}=B \cap e_{i}$ for $i=1,2$. Consider equivalence classes of $A$ determined by the cosets of the subgroup $H=\mathbb{Z} v_{2}+\mathbb{R} v_{1}$. One equivalence class of $A$ is $A_{1} \cup A_{2}$, and the rest are of the form $A \cap l$ for some 
line $l$ parallel to $\left[o, v_{1}\right]$, and intersecting int $[B]$. For such a line $l$ we claim that

$$
|(A \cap l)+B|>3|A \cap l|
$$

Indeed, if $\lambda>1$ then

$$
\left|(A \cap l)+B^{\prime}\right|=\left|(A \cap l) \cup\left((A \cap l)+v_{1}\right) \cup\left((A \cap l)+\left\{v_{2}, v_{3}\right\}\right)\right|>3|A \cap l| .
$$

If $\lambda=1$ then $\left|B_{1}\right|>2$ by our assumption, and we have $\left|(A \cap l)+B_{1}\right| \geq|A \cap l|+$ $\left|B_{1}\right|-1=|A \cap l|+2$. Hence,

$$
\begin{aligned}
\left|(A \cap l)+\left(B_{1} \cup B_{2}\right)\right| & =\left|(A \cap l)+B_{1}\right|+\left|(A \cap l)+B_{2}\right| \\
& \geq(|A \cap l|+2)+(2|A \cap l|-1)>3|A \cap l| .
\end{aligned}
$$

Since $\left|\left(A_{1} \cup A_{2}\right)+\left(B_{1} \cup B_{2}\right)\right| \geq 3\left|\left(A_{1} \cup A_{2}\right)\right|-3$ by Theorem 1 , it follows from (24) that $A=A_{1} \cup A_{2}$, and in turn $B \subset A$ implies that $B=B_{1} \cup B_{2}$. This shows that both $A$ and $B$ are contained in two parallel lines of the trapezoid. Therefore,

$$
|A+B|=\left|A_{1}+B_{1}\right|+\left|A_{2}+B_{2}\right|+\left|\left(A_{1}+B_{2}\right) \cup\left(A_{2}+B_{1}\right)\right|,
$$

where, by Proposition 8 (i), $\left|A_{i}+B_{i}\right| \geq 2\left|A_{i}\right|-1$ with equality if and only if $A_{i}$ is stable with respect to $B_{i}$. Moreover we have also $\left|\left(A_{1}+B_{2}\right) \cup\left(A_{2}+B_{1}\right)\right| \geq\left|A_{1}\right|+\left|A_{2}\right|-1$. Hence, it follows from $|A+B|=3|A|-3$ that there is equality in the three inequalities above, which implies

$$
A_{2} \subseteq A_{1}+v_{2} \text { and }\left(A_{1}+v_{2}\right) \cap\left[v_{2}, v_{3}\right] \subseteq A_{2}
$$

which together with the other two equalities imply (ii).

Lemma 27 If $(A, B)$ is a critical pair, and $[B]$ is a polygon, then $[B]$ has at most four vertices.

Proof We suppose that $P=[B]$ is a polygon of at least five vertices, and seek a contradiction. According to Theorem 23, we may assume that $P$ is a pentagon, and $B$ consists of the vertices of $P$. For any vertex $v$ of $P$, let $P_{v}$ be the convex hull of the other four vertices of $P$. It follows again by Theorem 23 that $\left(A \cap P_{v}, B \cap P_{v}\right)$ is a critical pair, as well, and hence Proposition 26 yields that $P_{v}$ is a trapezoid.

Since the sum of the angles of $P$ is $3 \pi$, there exists a side $f$ of $P$ such that the sum of the angles at the two endpoints is at least $\frac{6 \pi}{5}>\pi$. Let $e$ be the diagonal of $P$ not meeting $f$, and let $v$ be the vertex not in $e \cup f$. It follows that $P_{v}$ is a trapezoid where $e$ and $f$ are parallel, and $\ell(e)>\ell(f)$. We deduce from Proposition 26 that there exists $x \in A \cap e$ different from the endpoints of $e$.

Now let $w$ be an endpoint of $f$. Since $e$ is a diagonal of $P_{w}$, we have $x \in A \cap \operatorname{int} P_{w}$. However Proposition 26 (i) and (ii) applied to the pair $\left(A \cap P_{w}, B \cap P_{w}\right)$ shows that $A \cap \operatorname{int} P_{w}=\emptyset$, which is a contradiction. 
9 Critical Pairs $(A, B)$ where $[B]$ is an Iterated Pyramid Over a Simplex-Prism

Our first statement is a preparation for the proof of Lemma 29.

Lemma 28 If $A, B \subset \mathbb{R}^{d}, d \geq 2$, are finite such that $x+A \subset \operatorname{int}[B]$ for $x \in \mathbb{R}^{d}$, and $[B]$ is a d-dimensional simplex-prism, then

$$
|A+B|>(d+1)|A|
$$

Proof Let $\left[v_{0}, \ldots, v_{d-1}\right]$ and $\left[w_{0}, \ldots, w_{d-1}\right]$ be the facets of $[B]$ such that $\left[v_{i}, w_{i}\right]$ are the parallel vertical edges for $i=0, \ldots, d-1$, and

$$
\left\|v_{i}-w_{i}\right\| \leq\left\|v_{0}-w_{0}\right\| \text { for } i=1, \ldots, d-1
$$

We may assume that $v_{0}$ is the origin, and $A \subset \operatorname{int}[B]$. Let $\widetilde{B}=\left\{v_{0}, w_{0}, \ldots, v_{d-1}, w_{d-1}\right\}$ be the vertex set of $[B]$, and let $B^{\prime}=\left\{v_{1}, w_{1}, \ldots, v_{d-1}, w_{d-1}\right\}$.

We divide $A$ into equivalence classes according to the subgroup

$$
H=\mathbb{Z} v_{1}+\cdots+\mathbb{Z} v_{d-1}+\mathbb{R} w_{0},
$$

and hence adding $\widetilde{B} \subset H$ to different equivalence classes results in disjoint sets. As $A \subset \operatorname{int}[B]$ and $(25)$ yield that $A \subset(0,1) v_{1}+\cdots+(0,1) v_{d-1}+(0,1) w_{0}$, any equivalence class is of the form $A \cap l$ for a line $l$ parallel to $w_{0}$ and intersecting $A$, and a translate of $A \cap l$ is contained in $\left[v_{0}, w_{0}\right] \backslash\left\{v_{0}, w_{0}\right\}$. Thus Proposition 8 (ii) implies

$$
\left|(A \cap l)+\left\{v_{0}, w_{0}\right\}\right| \geq 2|A \cap l|
$$

We note that

$$
\text { the sets } l+v_{i}, i=0, \ldots, d-1 \text {, are pairwise disjoint, }
$$

therefore

$$
\left|(A \cap l)+B^{\prime}\right|=\sum_{i=1}^{d-1}\left|(A \cap l)+\left\{v_{i}, w_{i}\right\}\right| \geq \sum_{i=1}^{d-1}(|A \cap l|+1)>(d-1)|A \cap l|
$$

We conclude $|A+B| \geq|A+\widetilde{B}|>(d+1)|A|$ by (26), (27) and (28).

Combining Lemmas 24 and 28 yield the following.

Lemma 29 If $d>q \geq 2$ and $(A, B)$ is a critical pair such that $B$ spans $\mathbb{R}^{d}, A \subset$ $[B]$, and $[B]=\left[x_{1}, \ldots, x_{d-q}, P\right]$ for a q-dimensional simplex-prism $P$, then $A=$ $\left\{x_{1}, \ldots, x_{d-q}\right\} \cup(A \cap P)$.

It remains to describe the structure of $A$ and $B$ when $[B]$ is a simplex-prism. 
Proposition 30 Ifd $\geq 3$, and $(A, B)$ is a critical pair such that $[B]$ is a d-dimensional polytope projectively equivalent to a simplex-prism, then $B \subset A$, and

(i) the vertical edges of $[B]$ are parallel,

(ii) $A$ is contained in the vertical edges of $[B]$,

(iii) there exists a vertical vector $w \neq 0$ such that for each vertical edge $e, A \cap e$ can be partitioned into maximal arithmetic progressions of difference $w$ in e, one of them containing both endpoints of $e$, and this longest arithmetic progression contains $B \cap e$. In addition if e and $f$ are vertical edges, and $e+v \subset f$ in a way such that $e+v$ and $f$ share a common endpoint, then $(A \cap e)+v=A \cap(e+v)$.

Proof We have $B \subset A$ by Theorem 23 (i). Let $\left[v_{0}, \ldots, v_{d-1}\right]$ and $\left[w_{0}, \ldots, w_{d-1}\right]$ be the facets of $[B]$ such that $\left[v_{i}, w_{i}\right]$ are the vertical edges for $i=0, \ldots, d-1$.

For $0 \leq i<j \leq d-1$, it follows from Theorem 23 (iii) that $\left(A_{i j}, B_{i j}\right)$ is a critical pair for $A_{i j}=\left[v_{i}, w_{i}, v_{j}, w_{j}\right] \cap A$ and $B_{i j}=\left[v_{i}, w_{i}, v_{j}, w_{j}\right] \cap B$, therefore Proposition 26 yields that

$$
\left[v_{i}, w_{i}, v_{j}, w_{j}\right] \text { is a trapezoid, and }
$$

$A_{i j}$ and $B_{i j}$ satisfy the conditions of Proposition 26 (i) or (ii).

We verify (i) using an indirect argument. We suppose that the lines of the vertical edges meet at a point $p \in \mathbb{R}^{d}$, and seek a contradiction. We may assume that $w_{i} \in$ $\left[p, v_{i}\right]$ for $i=0, \ldots, d-1$. Since the pair composed of $A \cap\left[v_{0}, v_{1}, w_{1}, v_{2}, w_{2}\right]$ and $B \cap\left[v_{0}, v_{1}, w_{1}, v_{2}, w_{2}\right]$ is critical by Theorem 23 (iii), and $\left[v_{1}, w_{1}, v_{2}, w_{2}\right]$ is a trapezoid according to (29), Lemma 29 yields that

$$
A \cap\left[v_{0}, v_{1}\right]=\left\{v_{0}, v_{1}\right\}
$$

However $w_{i} \in\left[p, v_{i}\right]$ for $i=0,1$ and (29) yield that $\left[w_{0}, w_{1}\right]$ is parallel with and shorter than $\left[v_{0}, v_{1}\right]$. We deduce from Proposition 26 that $\left|A \cap\left[v_{0}, v_{1}\right]\right| \geq 3$, contradicting (31), and implying (i).

We prove (ii) again by contradiction, therefore we suppose that there exists an $x \in A$ not contained in the vertical edges.

According to the Charateodory theorem (see, e.g., Grünbaum [2]), if $x \in[X] \backslash X$ for $X \subset \mathbb{R}^{d}$, then $x \in\left[x_{0}, \ldots, x_{d}\right]$ for $x_{0}, \ldots, x_{d} \in X$. It follows that possibly after reindexing, there exists $m$ such that $1 \leq m \leq d,\left[x_{0}, \ldots, x_{m}\right]$ is an $m$-simplex, and

$x \in \operatorname{relint}\left[x_{0}, \ldots, x_{m}\right]=\left\{t_{0} x_{0}+\cdots+t_{m} x_{m}: t_{0}, \ldots, t_{m}>0\right.$ and $\left.t_{0}+\cdots+t_{m}=1\right\}$.

We deduce from (32) that there exists $1 \leq m \leq d$, and affinely independent vertices $x_{0}, \ldots, x_{m}$ of $[B]$ such that $x \in \operatorname{relint}\left[x_{0}, \ldots, x_{m}\right]$. Since an $m$-simplex has no two parallel edges, we may assume that $x_{0}=v_{0}$, and $w_{0} \notin\left\{x_{1}, \ldots, x_{m}\right\}$. In particular, $x \in$ $A \cap P$ for $P=\left[v_{0}, v_{1}, w_{1}, \ldots, v_{d-1}, w_{d-1}\right]$ where $Q=\left[v_{1}, w_{1}, \ldots, v_{d-1}, w_{d-1}\right]$ is a simplex-prism by (i). It follows from $x \in \operatorname{relint}\left[x_{0}, \ldots, x_{m}\right]$ that $x \neq v_{0}$ and $x \notin Q$. Since the pair $(A \cap P, B \cap P)$ is critical, this contradicts Lemma 29, and hence implies (ii).

The last property (iii) follows from (30) and Proposition 26. 


\section{Proof of Necessity in Theorem 5}

Let $(A, B)$ be a $k$-critical pair for some $k \geq 1$ with $\operatorname{dim}[B]=d$. In particular, $(A, B)$ a 1 -critical and $B$ is totally stackable by Theorem 23 . According to Theorem $12,[B]$ is a simplex, or an iterated pyramid over a polygon, or over (a projective deformation of) a simplex-prism. If $[B]$ is a simplex, then the characterization in Theorem 5 (i), (ii), (iv) and (vi) is achieved by Proposition 8 if $d=1$, and Proposition 25 if $d \geq 2$.

Therefore let $[B]$ be an iterated pyramid over $P$ with $\operatorname{dim} P=q$ where $P$ is polygon or a projective deformation of a simplex-prism. We may assume that $P$ is not a triangle. Since the pair ( $A \cap P, B \cap P$ ) is 1-critical by Theorem 23 (ii), Proposition 26 and 27 yield that if $P$ is a polygon, then it is a trapezoid. In addition, Proposition 30 yields that the vertical edges of $P$ are parallel even if $q \geq 3$.

We deduce from Lemma 29 that any point of $A$ is a vertex of $[B]$, or contained in $P$. Therefore we conclude Theorem 5 (iii) and (v) from Proposition 26 if $q=2$, and from Proposition 30 if $q \geq 3$.

Acknowledgments Part of the research was done during an FP7 Marie Curie Fellowship of the first name author at BarcelonaTech, whose hospitality is gratefully acknowledged. Károly J. Böröczky was supported by the FP7 IEF Grant GEOSUMSETS and OTKA Grant 109789. Francisco Santos was supported by the Spanish Ministry of Science (MICINN) through Grant MTM2011-22792. Oriol Serra was supported by the Spanish Ministry of Science (MICINN) under Project MTM2011-28800-C02-01, and by the Catalan Research Council under Grant 2009SGR1387.

\section{References}

1. Freiman, G.: Foundations of a Structural Theory of Set Addition. American Mathematical Society, Providence, RI (1973)

2. Grünbaum, B.: Convex Polytopes, 2nd edn. Springer, New York (2003)

3. Nill, B., Padrol, A.: The degree of point configurations: Ehrhart theory, Tverberg points and almost neighborly polytopes. Eur. J. Comb. arXiv:1209.5712

4. De Loera, J.A., Rambau, J., Santos, F.: Triangulations: Structures for Algorithms and Applications. Algorithms and Computation in Mathematics, vol. 25. Springer, Berlin (2010)

5. Matolcsi, M., Ruzsa, I.Z.: Sumsets and the convex hull. In: Chudnovsky, D., Chudnovsky, G. (eds.) Additive Number Theory: Festschrift in Honor of the Sixtieth Birthday of Melvyn B. Nathanson, pp. 221-227. Springer, New York (2010)

6. Ruzsa, I.Z.: Sumsets and structure. Combinatorial Number Theory and Additive Group Theory. Advanced Courses in Mathematics, CRM Barcelona, pp. 87-210. Birkhäuser, Basel (2009)

7. Stanchescu, Y.: On the simplest inverse theorem for sums of sets in several dimensions. Combinatorica 18, 139-149 (1998)

8. Stanley, R.P.: Cohen-Macaulay complexes. In: Aigner, M. (ed.) Higher Combinatorics. NATO Advanced Study Institute Series, pp. 51-62. Reidel, Dordrecht (1977)

9. Tao, T., Vu, V.: Additive Combinatorics. Cambridge University Press, Cambridge (2006)

10. Ziegler, G.M.: Lectures on Polytopes, revised edn. Springer, New York (1998) 